\title{
ANÁLISE DA DISTRIBUIÇÃO DE HEMOCOMPONENTES NA HEMORREDE DO DISTRITO FEDERAL
}

\author{
Diogo Jesus Cândido dos Reis \\ Ítalo Ricardo Santos Aleluia ${ }^{2}$ \\ Maísa Mônica Flores Martins ${ }^{3}$ \\ Elzo Pereira Pinto Junior ${ }^{4}$
}

REIS, D. J. C. dos; ALELUIA, I. R. S.; MARTINS, M. M. F.; PINTO JUNIOR, E. P. Análise da distribuição de hemocomponentes na hemorrede do Distrito Federal. Arq. Cienc. Saúde UNIPAR, Umuarama, v. 21, n. 2, p, 93-98, maio/ago. 2017.

\begin{abstract}
RESUMO: Este estudo analisou a distribuição de hemocomponentes nas agências transfusionais da Hemorrede do Distrito Federal, a partir do índice de descarte/expurgo, por vencimento, de bolsas de concentrado de hemácias. Trata-se de um estudo transversal com abordagem quantitativa e descritiva, a partir do Sistema de Gerenciamento do Ciclo do Sangue. Foram analisados descartes no período de 2013 a 2015 de 16 agências transfusionais. A análise estatística descreveu as frequências absolutas e relativas além do cálculo de medianas (Md), primeiro quartil (Q1) e terceiro quartil (Q3). Compõem o estudo as seguintes variáveis: total de bolsas de concentrado de hemácias; percentual de bolsas em cada serviço em relação ao total distribuído; quantidade de bolsas descartadas por vencimento; proporção de bolsas descartadas em relação ao total recebido em cada serviço. Alguns serviços indicaram um alto índice de descarte e outros apresentaram índices estáveis ou com tendência a discreta redução. O índice de descarte por vencimento não coincidiu com o quantitativo de bolsas distribuídas, revelando fortes possibilidades de descarte atrelado à má gestão do estoque ou a fragilidades na definição de critérios de solicitação das bolsas, desvinculando-as das necessidades reais e potenciais da população coberta pelas agências estudadas. É necessário aprimorar a gestão da distribuição e estoque, para racionalizar a oferta e as perdas de bolsas na maioria das agências transfusionais. Recomendam-se avaliações periódicas das demandas, inclusive nas agências que apresentaram altos índices de descarte persistentes, de modo a readequar a oferta e racionalizar a distribuição de bolsas.
\end{abstract}

PALAVRAS-CHAVE: Bancos de Sangue. Distribuição temporal. Serviço de hemoterapia.

\section{ANALYSIS OF BLOOD COMPONENTS AT THE BLOOD BANK NETWORK OF THE BRAZILIAN FEDERAL DISTRICT}

ABSTRACT: This study analyzed the distribution of blood components in transfusion agencies in the blood bank network of the Federal District from the disposal/purging index, by the expiration of concentrated RBC bags. This is a cross-sectional study from the Blood Cycle Management System with a quantitative and descriptive approach. It analyzes disposals from 16 blood transfusion centers in the period from 2013 to 2015. The statistical analysis described the absolute and relative frequencies as well as calculated medians (Md), first quartile (Q1) and third quartile (Q3). The study comprised the following variables: total concentrated RBC bags; percentage of bags in each service in relation to the total bags distributed; number of bags discarded due to expiration; proportion of discarded bags in relation to the total bags received in each service. Some services presented a high discard index while others had stable indexes or with a trend to discrete reduction. The discard rate due to expiration did not match the number of bags distributed, which strongly suggests disposal linked to bad inventory management or frailty in the definition of bag request criteria, dissociating them from the real and potential needs of the population attended by the blood banks studied. The inventory and distribution management must be improved in order to rationalize the offering and the losses of bags in most of the transfusion centers. A periodic assessment of the demands is recommended, including in agencies that present a high and persistent disposal rate, in order to adjust the supply and rationalize the distribution of bags.

KEYWORDS: Blood Banks. Hemotherapy Service. Temporal Distribution.

\section{Introdução}

A utilização de componentes produzidos a partir do sangue é uma prática corriqueira na medicina desde 1950, sendo essencial para prática biomédica nos dias atuais (SEKINE, 2008). Devido ao fato dos hemocomponentes serem produtos de oferta escassa, é preciso desenvolver abordagens que permitam a racionalização de seu uso e estocagem, para que seja possível atender a sua elevada demanda num cenário marcado pela ampliação dos serviços médico-hospitalares tanto na rede pública quanto na privada (GURGEL; CARMO, 2014).

O elevado índice de bolsas de sangue que são descartadas, seja por falhas na coleta ou no processamento, por vencimento do prazo de validade ou de descarte por testes de controle de qualidade, representam importante fator que dificulta o abastecimento de hemocomponentes. $\mathrm{O}$ controle inadequado dos estoques desses hemocomponentes em bancos de sangue pode produzir escassez desses produtos, o que gera prejuízos à assistência em saúde (GURGEL; CARMO, 2014).

A gestão adequada da distribuição, estocagem, uso e descarte de bolsas de sangue e hemocomponentes é importante para garantir o bom funcionamento de clínicas e hospitais que utilizam esses componentes para produzir o cuidado aos usuários dos seus serviços. Além disso, sob o ponto de vista financeiro, é preciso considerar o gasto com procedimentos que envolvem a produção e o uso de hemocompo-

DOI: https://doi.org/10.25110/arqsaude.v21i2.2017.6014

${ }^{1}$ Químico (UEGO). Fundação Hemocentro de Brasília. Especialista em Saúde Coletiva com Ênfase em Gestão de Serviços de Hemoterapia (ISC/UFBA). ${ }^{2}$ Fisioterapeuta. Mestre em Saúde Coletiva e Doutorando em Saúde Pública (ISC/UFBA). Professor Assistente da Universidade Federal do Oeste da Bahia. Endereço para correspondência: Av. José Bonifácio, JK, Ed. Olinda, n 1248, apto 102, Barreiras. Bahia. Brasil. Cep: $47800-305$.

Email: italoaleluia@yahoo.com.br

${ }^{3}$ Mestranda em Saúde Comunitária pelo Instituto de Saúde Coletiva da UFBA. Enfermeira pela Universidade do Estado da Bahia. Especialista em Gestão em Saúde (UNILAB). Professora da Universidade Católica do Salvador (UCSAL).

${ }^{4}$ Fisioterapeuta. Mestre em Saúde Coletiva (UECE). Doutorando em Saúde Pública (ISC/UFBA). Professor Substituto do Instituto de Saúde Coletiva (UFBA). 
nentes, já que dependem de recursos humanos especializados e tecnologias de alto custo. A boa administração dos hemocomponentes também envolve a fonte para sua obtenção, que é a doação voluntária de sangue, o que faz deste um insumo escasso, cuja segurança e racionalização são questões prioritárias (BRASIL, 2010).

São poucos os dados sobre descarte de bolsas de hemocomponentes encontrados na literatura. Ainda assim, nos Estados Unidos, em 2011, encontrou-se um percentual de descarte por vencimento em torno de 4,3\% dos hemocomponentes produzidos e distribuídos, ao passo que $0,8 \%$ são descartados por outras causas (HHS, 2013).

No Brasil, pesquisas revelam diferentes motivos de descarte de bolsas de sangue, dentre eles, destacam-se a inaptidão sorológica que corresponde de $10 \%$ a $20 \%$ das bolsas doadas (SALLES, 2004; FITARELLI; HORN, 2008); problemas técnico-operacionais dos serviços hemoterápicos; armazenamento excessivo e o descarte por vencimento (BRASIL, 2010; FONTE, 2004). Este último tem sido apontado como um dos principais fatores em vários estados, sobretudo quando se relaciona ao descarte de concentrado de hemácias $(\mathrm{CH})$ e bolsas de plaquetas randômicas (OLIVEIRA; BRITO, 2008).

Quando analisado o descarte de hemocomponentes no Paraná, entre 2010 a 2012, verificou-se que $80 \%$ do total da produção foi descarta em função do vencimento de bolsas de $\mathrm{CH}$ com sorologia já realizada e apta para o uso; devolução de bolsas de $\mathrm{CH}$ sem condição de uso; rompimento de bolsas e pela concentração de hemoglobina inferior a $45 \mathrm{~g} /$ dL. No Rio Grande do Norte, somente o descarte por vencimento deteve $51 \%$ do total de bolsas de $\mathrm{CH}$ descartadas no período estudado compreendido entre 2004 a 2007 (MOURA 2013; OLIVEIRA; BRITO, 2008).

No estado do Rio Grande do Sul, Fonte (2004) apontou importantes percentuais de descarte de concentrado de hemácia, por vencimento. Entretanto, cabe assinalar que houve diferenças significativas entre serviços públicos e privados, com maior predominância de descarte neste último.

Experiências bem sucedidas de gestão da qualidade e distribuição destacaram que a qualificação contínua dos profissionais envolvidos nos processos de gestão de bancos de sangue e hemoderivados contribuíram significativamente para reduzir o descarte de hemocomponentes, padronizando processos e garantindo o monitoramento de variações atípicas no processamento desses insumos (OLIVEIRA; BRITO, 2008; GIRARDELLO, 2015).

Ainda são escassos na literatura nacional estudos que analisem o descarte de hemocomponentes. A identificação de dados que demonstrem a magnitude desse problema pode interferir na gestão dos estoques de hemocomponentes, cujo gerenciamento ineficiente produz escassez desse insumo, prejudicando a assistência ao usuário dos serviços de saúde. Dessa forma, o objetivo do presente estudo foi analisar a distribuição de hemocomponentes nas agências transfusionais da Hemorrede do Distrito Federal, a partir do índice de descarte/expurgo, por vencimento, de bolsas de $\mathrm{CH}$.

\section{Metodologia}

Trata-se de um estudo transversal com abordagem quantitativa e descritiva. Os dados utilizados nesse estudo derivaram de registros do banco informatizado da Fundação Hemocentro de Brasília - FHB, especificamente do Sistema de Gerenciamento do Ciclo do Sangue da FHB - SistHemo. Esse banco de dados contém todas as informações relativas à coleta, ao processamento, ao estoque, à distribuição, à transfusão e ao expurgo/descarte dos hemocomponentes encaminhados às unidades de saúde do Distrito Federal.

A Fundação Hemocentro de Brasília (FHB) é o hemocentro de referência que abastece os serviços públicos e conveniados de saúde no DF e, que, portanto, apresenta dados sobre coleta e distribuição de hemocomponentes. A FHB tem como objetivo principal implementar ações de captação, coleta, processamento e distribuição de sangue para uma população de 2,85 milhões habitantes, em um território de $5.802 \mathrm{~km}$, onde contém agências transfusionais de hospitais públicos (12), em regime de parceria público-privada (três), regime militar (um) e privados conveniados (cinco) (BRASIL, 2014).

A hemorrede do DF é constituída de um hemocentro de referência, a Fundação Hemocentro de Brasília, responsável pela distribuição de hemocomponentes por ela produzidos e pela manutenção de estoques em 16 agências transfusionais de hospitais públicos e conveniados ao Sistema Único de Saúde, sendo um hospital de base, 10 hospitais regionais, uma fundação universitária, um hospital universitário, dois hospitais especializados e um hospital das forças armadas, as quais participaram desse estudo.

Esse estudo analisou as bolsas de $\mathrm{CH}$ como proxy dos hemocomponentes, dada sua representatividade em mais de $60 \%$ dos procedimentos de transfusão e uso realizados em ambulatórios de sangue, sendo, portanto, o hemocomponente mais utilizado no País e no Distrito Federal conforme avaliações do Ministério da Saúde (BRASIL, 2014).

Os dados desta pesquisa são relacionados à distribuição e ao descarte das bolsas de $\mathrm{CH}$ no período de 2013 a 2015, haja vista que todas as agências transfusionais incluídas nesse estudo passaram a ser informatizadas em 2012, sendo este ano inicial caracterizado como um período de transição dos registros da distribuição e do descarte.

Para compor os resultados desta pesquisa foram incluídas agências transfusionais que mantivessem estoques regulares no Estado e a existência de dados completos no sistema de informação, para o período definido na pesquisa, totalizando 16 instituições que recebem bolsas por meio da distribuição de rotina. Para preservar as instituições, optou-se por não apresentar seus nomes, tendo sido substituídos por letras.

As variáveis que compõem esse estudo foram as seguintes: total de bolsas de $\mathrm{CH}$; percentual de $\mathrm{CH}$ em cada serviço em relação ao total de bolsas distribuídas; quantidade de bolsas descartadas por vencimento; proporção de bolsas descartadas em relação ao total recebido em cada serviço.

A análise estatística contou com a descrição das frequências absolutas e relativas e cálculo de medianas $(\mathrm{Md})$, primeiro quartil (Q1) e terceiro quartil (Q3). Para apresentação da distribuição dos dados optou-se pela construção de gráficos do tipo Box plot. Os dados foram tabulados em planilhas de texto Excel, da Microsoft, e analisados no programa Stata, versão 12, da IBM.

Considerando que esse estudo trabalhou com dados secundários, em obediência à resolução 466/2012 do Conse- 
lho Nacional de Ética em Pesquisa, obteve-se a autorização para utilização do banco de dados, por meio da Carta de Anuência Institucional da Coordenação Estadual da Hemorrede do Distrito Federal.

\section{Resultados}

De 2013 a 2015, foram distribuídas 131.124 bolsas de concentrado de hemácias nos 16 serviços que compõem a hemorrede de Brasília. No mesmo período, foram descartadas por vencimento 7.088 bolsas, o que representou $5,4 \%$ do total de bolsas fornecidas.

A mediana de bolsas recebidas por cada serviço de hemoterapia foi $6.468,5(\mathrm{Q} 1=4.160,5$; Q3=8.667,0), variando de 2.575 até 34.614 bolsas recebidas (Gráfico 1). Em relação ao descarte de bolsas de concentrado de hemácias, a mediana foi 415,5 (Q1=254,5; Q3=668), com variação de 45 até 934 bolsas não aproveitadas (Gráfico 2).

Gráfico 1: Quantidade de bolsas de $\mathrm{CH}$ recebidas pelos serviços de hemoterapia que compõem a hemorrede de Brasília, 2013-2015.

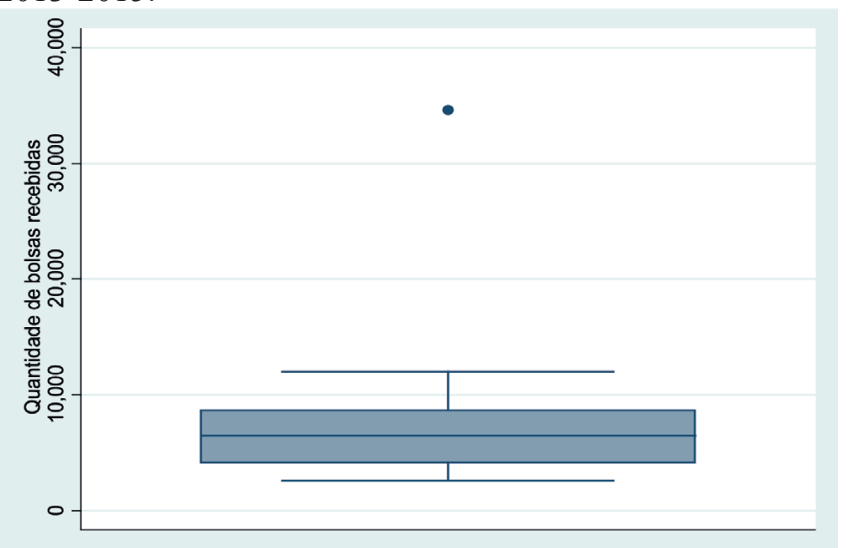

Gráfico 2: Quantidade de bolsas de $\mathrm{CH}$ descartadas pelos serviços de hemoterapia que compõem a hemorrede de Brasília, 2013-2015.

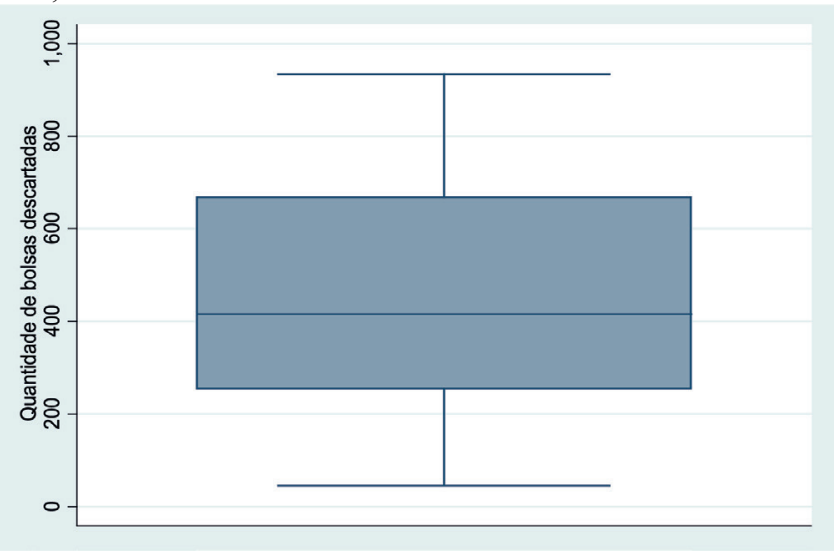

A análise do recebimento das bolsas de concentrados de hemácias evidenciou que na hemorrede de Brasília havia um serviço que recebeu por $26,4 \%$ de todas bolsas (serviço A), valor muito acima do encontrado nos demais serviços. Por outro lado, os serviços de menor porte (serviços O e L) foram aqueles que tiveram maior proporção de bolsas descartadas, figurando nas primeiras posições do ranking de descarte (Quadro 1).

Quadro 1: Ranking de descarte de bolsas de concentrado de hemácias na hemorrede de Brasília, 2013-2015.

\begin{tabular}{|c|c|c|c|c|c|c|}
\hline Serviços & $\begin{array}{c}\text { Quantidade de } \\
\text { bolsas recebidas }\end{array}$ & $\begin{array}{c}\text { Proporção de } \\
\text { bolsas recebidas }\end{array}$ & $\begin{array}{c}\text { Ranking de } \\
\text { bolsas recebidas }\end{array}$ & $\begin{array}{c}\text { Quantidade } \\
\text { de bolsas } \\
\text { descartadas }\end{array}$ & $\begin{array}{c}\text { Proporção de } \\
\text { descarte }\end{array}$ & $\begin{array}{c}\text { Ranking } \\
\text { de bolsas } \\
\text { descartadas }\end{array}$ \\
\hline & 34.614 & $26,4 \%$ & 1 & 675 & $2,0 \%$ & 14 \\
\hline B & 12.016 & $9,2 \%$ & 2 & 732 & $6,1 \%$ & 8 \\
\hline C & 10.957 & $8,4 \%$ & 3 & 575 & $3,2 \%$ & 10 \\
\hline D & 8.831 & $6,7 \%$ & 4 & 339 & $3,8 \%$ & 12 \\
\hline E & 8.503 & $6,5 \%$ & 5 & 233 & $2,7 \%$ & 13 \\
\hline F & 8.024 & $6,1 \%$ & 6 & 45 & $7,6 \%$ & 16 \\
\hline G & 8.006 & $6,1 \%$ & 7 & 730 & $9,1 \%$ & 7 \\
\hline H & 6.489 & $4,9 \%$ & 8 & 934 & $14,4 \%$ & 3 \\
\hline I & 6.448 & $4,9 \%$ & 9 & 60 & $0,9 \%$ & 15 \\
\hline J & 6.051 & $4,6 \%$ & 10 & 276 & $4,6 \%$ & 11 \\
\hline K & 4.504 & $3,4 \%$ & 11 & 498 & $11,1 \%$ & 4 \\
\hline L & 4.302 & $3,3 \%$ & 12 & 661 & $15,4 \%$ & 2 \\
\hline M & 4.019 & $3,1 \%$ & 13 & 411 & $10,2 \%$ & 6 \\
\hline N & 3.272 & $2,5 \%$ & 14 & 356 & $10,9 \%$ & 5 \\
\hline O & 2.603 & $2,0 \%$ & 15 & 420 & $16,1 \%$ & 5 \\
\hline P & 2.575 & $2,0 \%$ & 16 & 143 & $5,6 \%$ & 1 \\
\hline Total & 131.214 & $100,0 \%$ & - & 7.088 & $5,4 \%$ & 9 \\
\hline Sis & & & & - \\
\hline
\end{tabular}

Fonte: SistHemo-DF 


\section{Discussão}

A análise da distribuição e do descarte de $\mathrm{CH}$ evidenciou, de modo geral, baixa proporção de descarte desses hemocomponentes. Entretanto, os dados permitiram observar que essa proporção de descarte foi muito maior em serviços de menor porte, o que aponta problemas no manejo desses insumos quando comparados à serviços de maior porte.

Dados do Ministério da Saúde apontaram que de todos os procedimentos de transfusão no SUS e em unidades privadas, tanto ambulatorial quanto hospitalar, realizados em todo o País, desde 2012, já envolviam predominantemente o uso de concentrado de hemácias, correspondendo a quase $60 \%$ de seu total, o que ratifica a representatividade deste hemocomponente na utilização de ações hemoterápicas no país (BRASIL, 2014).

Nos anos de 2010 a 2012, no Distrito Federal, apesar da inexistência de registros de coletas no setor privado, o percentual dos procedimentos de transfusão ambulatorial e hospitalar, por tipo de hemocomponentes, no mesmo período, apresentou uma frequência de $70 \%$ de procedimentos envolvendo a utilização de concentrados de hemácias (BRASIL, 2014).

A Organização Mundial da Saúde preconiza como padrão um percentual de 5\% no índice de descarte de bolsas de $\mathrm{CH}$, por vencimento (FONTE, 2004). Quando comparado esse padrão com os resultados encontrados nessa pesquisa, parece-nos evidente que uma minoria das unidades transfusionais do DF realizam uma gestão racional da distribuição e estocagem do sangue, haja vista que a proporção de descarte durante o período analisado foi superior a $5 \%$ na maior parte dos serviços investigados.

Estudos no âmbito internacional encontraram situação oposta à evidenciada no DF. Territórios localizados na América do Norte, América Central e Ásia apresentam percentuais de descarte por vencimento, predominantemente da ordem de 1\%, a 4\%. Em média os Estados Unidos, Índia e Nicarágua corresponderam a índices de descarte em torno de 2,5\%, 1\% e 3,89\%, respectivamente (HHS, 2013; SURESH et al., 2015).

Dentre os motivos explicativos para o descarte por vencimento no contexto internacional, destacou-se a existência de falhas na execução da gestão do estoque, em que não havia controle baseado na data de entrada e saída dos hemocomponentes (SURESH et. al., 2015). É possível que este fato também possa explicar a elevação de índices de descarte em boa parte dos serviços analisados nesse estudo. A presente questão ratifica a importância do monitoramento regular do estoque.

Outros motivos de descarte de hemocomponentes também envolvem a inaptidão sorológica. Um estudo revelou que os principais marcadores do descarte estavam atrelados ao anti-HBc (1,67\%), seguido do VDRL (0,65\%), HBsAg $(0,18 \%)$, anti-HCV $(0,12 \%)$, Chagas $(0,06 \%)$, anti-HIV $(0,05 \%)$ e anti-HTLV $(0,02 \%)$ (MICHELIN et al., 2008).

Segundo Repolês (2014) os resultados falso-positivos e inconclusivos podem corroborar para descarte desnecessário de bolsas de sangue, o que justifica a necessidade de métodos capazes de distinguir com segurança resultados verdadeiros positivos dos falsos, já que essa situação somada ao descarte por vencimento prejudicam a gestão de estoque nos hemocentros.

No Brasil, investigações apontaram bastante heterogeneidade no índice descarte de bolsas de $\mathrm{CH}$, por vencimento. Em Porto Alegre, por exemplo, esse índice variou entre $4,35 \%$ a $61,46 \%$, sendo o valor máximo muito superior ao evidenciado no presente estudo (FONTE, 2004). Já no Paraná, comparando-se os anos de 2010 a 2012, esse tipo de descarte variou entre $1,76 \%$ a $1,85 \%$ revelando valores bem abaixo dos índices encontrados no DF (MOURA, 2013).

Diferentemente das evidências apontadas nesse estudo, encontrou-se que o volume excedente das bolsas de $\mathrm{CH}$ se configurou como descarte no Rio Grande do Norte (OLIVEIRA, 2008). Em um município de São Paulo, 99,13\% das bolsas de $\mathrm{CH}$ eram utilizadas até a data de vencimento, embora o principal motivo de descarte das bolsas de plaquetas, por vencimento, correspondesse a $53,17 \%$ e o índice de descarte geral de hemocomponentes ficou aquém do estipulado (8,53\%) (PALLUDETO, 2015).

No período analisado ficou evidente que houve uma relação inversa entre a totalidade de bolsas distribuídas e descartadas. Isso porque no período inicial e final analisado houve aumento no total de bolsas distribuídas e a redução do descarte em torno para alguns serviços; e houve redução na quantidade de bolsas distribuídas de aproximadamente seguida do aumento na quantidade de descarte por vencimento para outros. É possível que esse quadro tenha relação com a informatização da Hemorrede, em 2012, o que permitiu uma melhora no gerenciamento da distribuição e dos estoques nas agências transfusionais.

Verificou-se que no período analisado alguns serviços lideraram o ranking do índice de descarte e outros apresentaram índices estáveis ou com tendência a discreta redução. Nesse sentido, cabe assinalar que a possibilidade dessa situação está atrelada às características organizacionais das agências transfusionais.

Corroborando com isso, Fonte (2004) evidenciou que auditorias em agências transfusionais constataram solicitações de hemocomponentes para realização de cirurgias que ocorrem sem a necessidade de transfusão, o que contribuiu para estoques excessivos e significativos índices de descarte por prazo de validade vencido.

Por outro lado, o monitoramento das perdas de bolsas de sangue, por hemocentros, parece ser um problema persistente em alguns estados do Brasil, a exemplo do Rio Grande do Sul (FONTE, 2004). Esse fato, assim como as informações evidenciadas no DF, justificam a implantação de mecanismos mais eficientes para controlar sistematicamente tais perdas e fortalecem a afirmativa de autores que defendem a implantação de estratégias para redução do descarte (FERREIRA et al., 2013).

Constatou-se que o índice de descarte provavelmente não possuiu relação com a quantidade de bolsas de $\mathrm{CH}$ distribuídas. Esse fato remonta para problemas na otimização do uso dos hemocomponentes, pelas agências transfusionais, observando o prazo de validade, em relação a outras que apresentam um índice alto descarte. Para analisar essa situação é conveniente levar em consideração o fator geográfico e o contingente populacional coberto por essas agências, haja vista que alguns dos serviços que apresentam maior percentual de descarte são aqueles mais distantes dos grandes centros urbanos e que atendem a cidades de pequeno porte. 
Outra questão diz respeito à influência do maior ou menor prazo de validade atrelado ao hemocomponente, que a depender da data de distribuição e da relação de oferta e demanda nas agências poderá determinar diretamente o descarte por vencimento.

Considerando o exposto, parece que o uso de alternativas de otimização do descarte como a coleta por aférese e o monitoramento da validade e da redistribuição antes da expiração do prazo, para um serviço que esteja demandando o hemocomponente, tem sido amplamente defendida pela literatura (FERREIRA et al., 2013; FONTE, 2004).

Estudos ressaltam que os descartes de bolsas de sangue coletados levam a consideráveis perdas sociais e financeiras e à elevação dos custos na manutenção dos sistemas de coletas e produção de hemocomponentes. Isso justifica a relevância de campanhas educativas que envolvam desde os aspectos responsáveis pela inaptidão sorológica àqueles que abarcam medidas de segurança transfusional; e da realização de novas pesquisas relativas à estrutura física, organização do processo de trabalho das equipes técnicas e dos métodos de gestão de estoque adotados, para identificação marcadores que diferenciam agências com menores e maiores índices de descarte por vencimento (PALLUDETO, 2015; (MICHELIN et al., 2008).

De acordo com outros autores, o descarte de hemocomponentes representa não apenas a prevalência de inaptidões sorológicas na população de doadores, mas, principalmente, a qualidade do sangue e hemocomponentes disponibilizados para transfusão (FERREIRA, 2012; ROHR et al., 2012).

As falhas durante a coleta e processamento, prazo de validade vencido ou rejeição após os testes de controle de qualidade podem levar a baixa taxa de oferta e ao alto índice de descarte de bolsas de sangue. Isso reflete sobre a oferta e oferece risco para a vida dos usuários. Por sua vez, a falta de controle do estoque dos bancos de sangue pode causar diversos prejuízos, inclusive a escassez de sangue e o desperdício.

\section{Conclusão}

O presente estudo teve como objetivo analisar a gestão da distribuição de hemocomponentes nos serviços de transfusão de sangue da Hemorrede do Distrito Federal, por meio do índice de descarte/expurgo por vencimento de bolsas de concentrado de hemácias.

Os dados podem apontar que o descarte por vencimento não coincide com o quantitativo de bolsas distribuídas, revelando que há fortes possibilidades desse descarte está atrelado à má gestão ou solicitação de estoque desvinculado das necessidades reais e potenciais das agências estudadas. Por outro lado, agências transfusionais com baixos índices de descarte por vencimento podem denotar boas práticas de gestão de estoque não realizadas pelas unidades de saúde que apresentam altos índices.

Os resultados aqui delineados apontaram para a necessidade de fortalecimento da gestão da distribuição e estoque, a fim de racionalizar a oferta e minimizar as perdas de bolsas na maioria das agências transfusionais. Recomendam-se avaliações periódicas das demandas, inclusive nas agências que apresentaram altos índices de descarte persistentes, de modo a readequar a oferta e racionalizar a distribuição de bolsas.

\section{Referências}

BRASIL. Ministério da Saúde. Guia para o uso de hemocomponentes. Brasília: Ministério da Saúde, 2010.

Ministério da Saúde. Caderno de informação: sangue e hemoderivados. 7. ed. Brasília: Ministério da saúde, 2014.

CRELIER, F.; FIGUEIREDO, S.; LEONARDO, H. Modelagem e Gestão dos Estoques de Sangue do HEMORIO. Rio de Janeiro: UFRJ. Escola Politécnica; (Trabalho de Conclusão de Curso). 2016; 72 p.

FERREIRA, D. M.; GRIZA, D.; SISTI, E. Análise dos aspectos epidemiológicos, hematológicos e sorológicos presentes em doadores de sangue do Hemocentro Regional de Cruz Alta. Rev. bras. anal. clin; v. 44, n. (1), p. 10-14, 2012.

FERREIRA, A. A. et al. Segurança transfusional: avaliação da produção, distribuição e eficácia transfusional do concentrado de plaquetas na Hemorrede do Estado de Minas Gerais. GERAIS: Revista de Saúde Pública do SUS/MG, Minas Gerais, v. 1, n. 1, p. 143-144, 2013.

FITARELLI, D. B.; HORN, J. F. Descarte de bolsas de sangue devido à reatividade para doença de Chagas em um laboratório de triagem sorológica de doadores em Porto Alegre-RS. Rev. Bras. Hematol. Hemoter. São Paulo, v.31, n. 5, 2009.

FONTE, C. A. G. Como otimizar a utilização do sangue no estado do Rio Grande do Sul. Trabalho de conclusão de curso - Programa de Desenvolvimento da Gestão em Saúde - PDG. Universidade Federal do Rio Grande do Sul. 2004.

GIRARDELLO, M. Gestão de Qualidade em Saúde: o caso do Hemocentro de Caxias do Sul - RS. 2015. 46f. Monografia (Especialização) - Escola de Administração, Universidade Federal do Rio Grande do Sul, Novo Hamburgo, 2015.

GURGEL, J. L. M.; CARMO, B. B. T. do. Dimensionamento do estoque de derivados de sangue em um hemocentro do Brasil baseado em um modelo de gestão de estoques e previsão de demanda. Revista Produção Online, Florianópolis, v.14, n. 1, p. 264-293, jan./mar. 2014.

HHS - THE UNITED STATES DEPARTMENT OF HEALTH AND HUMAN SERVICES. The 2011 national blood collection and utilizationsurvey report - NBCUS. USA. 2013.

MOURA, E. L. de. Identificação dos fatores de descarte de bolsas na produção de hemocomponentes no hemocentro coordenador da rede HEMEPAR. Curitiba. 
2013. Projeto Técnico. MBA em Gestão da Qualidade. Universidade Federal do Paraná, 2013.

OLIVEIRA, L. M. de. A utilização do controle estatístico do processo para o monitoramento do sangue: estudo de caso no Hemonorte - RN. Natal, 2008. Dissertação (Mestrado em Ciências em Engenharia de Produção) Programa de Engenharia de Produção, Universidade Federal do Rio Grande do Norte, 2008.

OLIVEIRA, M. de F. R. de; BRITO, M. do S. de F. Experiência de gestão no Hemope Caruaru de julho de 2004 a junho de 2007. 2008. 36f. Monografia (Especialização) Centro de Pesquisas Aggeu Magalhães, Fundação Oswaldo Cruz, Recife, 2008.

PALUDETTO, N. O. Implantação de gestão da qualidade no serviço de hemoterapia em um hospital público do Estado de São Paulo. Universidade Estadual Paulista, Campus de Botucatu. [ Dissertação de Mestrado]. Mestrado em Pesquisa e Desenvolvimento - Biotecnologia Médica. 2015.

MICHELIN. R. D. et al. Prevalência de marcadores sorológicos no Banco de Sangue de Caxias do Sul, RS. Rev. panam. infectol; v. 10, n. 3, p. 32-35, jul.-set. 2008.

REPOLÊS, L. C. Avaliação do método confirmatório FC-TRIPLEX-IgG1 no esclarecimento diagnóstico e na monitoração sorológica para doença de Chagas em bancos de sangue. Belo Horizonte; 2014. 113 p.

ROHR, I. J.; BOFF, D.; LUNKES, D. S. Perfil dos candidates inaptos para doação de sangue no serviço de hemoterapia do hospital Santo Ângelo, RS, Brasil. Rev. Patol. Trop., v. 41, n. 1, p. 27-35, 2012.

SALLES, N. A. et al. Descarte de bolsas de sangue e prevalência de doenças infecciosas em doadores de sangue da Fundação Pró-Sangue/Hemocentro de São Paulo.

Rev Panam Salud Publica/Pan Am J Public Health, Washington, v. 13, n. 2-3, 2003

SEKINE, L. et al. Análise do perfil de solicitações para transfusão de hemocomponentes no Hospital de Clínicas de Porto Alegre no ano de 2005. Rev. bras. hematol. hemoter. p. 208-212. 2008.

SURESH B. et al. Reasons for discarding whole blood and its components in a tertiary care teaching hospital blood bank in South India. J Clin Sci Res. p. 213-219, 2015. 